\title{
Effects of microcystin-LR on gut microflora in different gut regions of mice
}

\author{
Jing Chen ${ }^{1,2}$, Ping Xie ${ }^{1}$, Juan Lin ${ }^{1,2}$, Jun He${ }^{1}$, Cheng Zeng ${ }^{3}$ and Jun Chen ${ }^{1}$ \\ 'Donghu Experimental Station of Lake Ecosystems, State Key Laboratory of Freshwater Ecology and Biotechnology \\ of China, Institute of Hydrobiology, Chinese Academy of Sciences, Donghu South Road 7, Wuhan 430072, China \\ ${ }^{2}$ University of Chinese Academy of Sciences, Beijing 100049, China \\ ${ }^{3}$ College of Fisheries, Huazhong Agricultural University, Wuhan 430070, China
}

(Received March 5, 2015; Accepted May 8, 2015)

\begin{abstract}
To reveal the toxicological effects of the hepatotoxic microcystin-leucine arginine (MC-LR) on gut microbial community composition in different gut regions, we conducted a subchronic exposure of BALB/c mice to MC-LR via intragastric administration. Denaturing gradient gel electrophoresis (DGGE) was employed to profile the shifts of microbes after MC-LR treatment in the jejuno-ileum, caecum and colon. DGGE profiles analysis showed that MC-LR increased the microbial species richness (number of microbial bands) in the caecum and colon as well as microbial diversity (ShannonWiener index) in the caecum. The cluster analysis of DGGE profiles indicated that the microbial structures in the caecum and colon shifted significantly after MC-LR treatment, while that in the jejuno-ileum did not. All the relatively decreased gut microbes belonged to Clostridia in the Firmicutes phylum, and most of them were Lachnospiraceae. The increased ones derived from a variety of microbes including species from Porphyromonadaceae and Prevotellaceae in the Bacteroidetes phylum, as well as Lachnospiraceae and Ruminococcaceae in the Firmicutes phylum, and among which, the increase of Barnesiella in Porphyromonadaceae was most remarkable. In conclusion, subchronic exposure to MC-LR could disturb the balance of gut microbes in mice, and its toxicological effects varied between the jejuno-ileum and the other two gut regions.
\end{abstract}

Key words: MC-LR, Gut microbial community, Mice, Intestine

\section{INTRODUCTION}

Microcystins (MCs), a family of toxic monocyclic heptapeptides produced by cyanobacteria, are a worldwide threat to humans and animals due to their presence in both drinking and recreational waters (Codd, 2000). So far, numerous incidents of MCs intoxication have been reported in animals and humans, among which the most serious one is the deaths of over 50 hemodialysis patients in Caruaru, Brazil in 1996 (Pouria et al., 1998; Metcalf et al., 2001; Lürling and Faassen, 2013). MCs have more than 100 different structural analogs and the most common and toxic one is microcystin-leucine-arginine (MC-LR) (Zhang et al., 2009; Yan et al., 2012). It is well known that MC-LR has potent hepatotoxicity and tumor-promoting activity (Nishiwaki-Matsushima et al., 1992; Li et al., 2012), which targets the liver and can induce various liver diseases including hepatitis and carcinoma (Chen et al., 2005). The toxicity mechanism of MC-LR is mainly based on its inhibition of protein phosphatases PP1 and PP2A, leading to hyperphosphorylation of cytosolic and cytoskeletal proteins and disruption of cell cytoskeletal components (Fastner et al., 2002; $\mathrm{Li}$ and Xie, 2009). Moreover, it has been widely revealed that MCs could induce the production of reactive oxygen species (ROS), causing oxidative damage and apoptosis (Jiao et al., 2014a).

MC-LR is not cell permeant; it requires uptake via the bile acid transport system (enterohepatic cycling) present in hepatocytes and cells lining the small intestine (Runnegar et al., 1991; Dawson, 1998). Ito et al. (2000) found that orally taken MC-LR was mainly absorbed in the small intestine. Via portal vein, the intestine absorbed MC-LR as well as metabolic products of gut microflora could be delivered from gut to liver and other organs by blood (Faber et al., 2003; Goel et al., 2014). Gastroin-

Correspondence: Jun Chen (E-mail: chenjun@ihb.ac.cn) 
testinal ailments induced by MCs have been reported in fish, mice and humans (Humpage et al., 2000; Botha et al., 2004; Messori et al., 2013). For instance, MC-LR can induce cell apoptosis in the intestinal tract in mice (Botha et al., 2004), and inhibit activities of the digestive enzymes from intestinal mucosa and cause intestinal lesions (Liu and Xie, 2014).

In the past several years, increasing attention has been given to the gastrointestinal tract, as trillions of microbes reside there and in many ways these microbes have been implicated in host health and disease etiology (Hooper et al., 2002; Bates et al., 2007; Loh and Blaut, 2012). The intestine is divided into various well-defined anatomical regions, namely the duodenum, jejunum, ileum (belonging to the small intestine), caecum and colon (belonging to the large intestine). Meanwhile, the gut microbes are not distributed randomly throughout the intestinal tract but instead vary with intestinal topography to adapt to the environments or facilitate the functions of different gut regions (Mackie et al., 1999; Tian et al., 2011; Liu et al., 2012). The topographical metabolic variations of gut microbes have also been revealed both in vivo (Tian et al., 2011; Druart et al., 2014) and in vitro (Jiao et al., 2014a, 2014b). As one of the most common pollutants, MC-LR could have various effects on the gut microbes residing in different gut regions.

In order to evaluate the toxicological effects of the MCLR on gut microbial community in different gut regions, we gave $\mathrm{BALB} / \mathrm{c}$ mice a sub-chronic exposure to $\mathrm{MC}$ LR through intragastric administration, and employed denaturing gradient gel electrophoresis (DGGE) method to study the variations of microflora in mice jejuno-ileum, caecum and colon after MC-LR treatment. This work could provide primary information of gut microflora concerning MC-LR toxicity assessment.

\section{MATERIALS AND METHODS}

\section{Extraction and purification of MC-LR}

Microcystins were extracted and purified from freezedried surface blooms collected from Lake Dianchi in Yunnan, China according to Li and Xie (2009). MCLR was separated by semi-performance preparative liquid chromatography system (Waters 600E, Milford, MA, USA) and a reverse-phase high-performance liquid chromatography (HPLC, LC-20A, Shimadzu, Kyoto, Japan). The purity of MC-LR was over $95 \%$ (the remainder was mainly comprised of pigment) and the identity and quantification of MC-LR were confirmed by liquid chromatography-electrospray ionization mass spectrometry (LC-ESI/MS, Thermo Electron, Waltham, MA, USA) method described previously (Qin et al., 2012). MC-LR standards were obtained from Sigma-Aldrich Ltd (St. Louis, MO, USA).

\section{Animal experiment}

A total of twelve six-week-old male BALB/c mice (Experimental Animal Central of Beijing, China) with similar weights $(22.46 \pm 0.93 \mathrm{~g})$ were raised in a specific pathogen-free environment at the animal facilities of Wuhan Institute of Virology, Chinese Academy of Science, under controlled conditions (temperature, 20-22 ${ }^{\circ} \mathrm{C}$; relative humidity, $40-60 \%$; day-night light cycle, 12-12 hr). The mice had free access to sterile food and water. The food and water were refreshed every two days, and wood-chip bedding and the cages were refreshed every week in the experimental period. Body weight was measured every week. After two week's accommodation, the mice were randomly separated into two groups: the treated group $(n=6)$ which was given MC-LR at the dose of $200 \mu \mathrm{g} / \mathrm{kg}$ body weight by intragastric administration and the control group $(n=6)$ which was given $0.9 \%$ sterile saline. The treatment was given every 2 days for 3 months (45 times in total). All the animal experiments were conducted according to the National Institutes of Health Guide for the Care and Use of Laboratory Animals (NIH Publication No.8023).

\section{Sample collection and total DNA extraction}

Three months later, the mice were humanely sacrificed. The jejuno-ileum, caecum and colon were separated immediately according to Puhl et al. (2012). Samples of the digesta were quickly removed from the lumen of each intestinal region into $1.5 \mathrm{~mL}$ sterile Eppendorf tubes $(\mathrm{Wu}$ et al., 2014), and immediately frozen with liquid nitrogen before being stored at $-80^{\circ} \mathrm{C}$ (Druart et al., 2014). Bacterial DNA was extracted from equal amounts of digesta samples using the E.Z.N.A. Stool DNA Kit (Omega Biotek Inc., Norcross, GA, USA).

\section{S rRNA gene amplification and DGGE}

The V3 region of $16 \mathrm{~S}$ rRNA gene of gut microbes was amplified with the primers $341 \mathrm{~F}-\mathrm{GC}$ (5'-GC clampCCT ACG GGA GGC AGC AG-3') and 518R (5'-ATT ACC GCG GCT GCT GG-3'). Each $50 \mu \mathrm{L}$ PCR reaction mixture contained $1 \times$ PCR Buffer $\left(1.5 \mathrm{mM} \mathrm{Mg}^{2+}\right.$ plus $)$, $0.2 \mathrm{mM}$ of each dNTP, $10 \mathrm{p} \mathrm{mol}$ of each primer, $1.25 \mathrm{U}$ of Taq DNA polymerase, and $60 \mathrm{ng}$ of DNA template. A touchdown PCR amplification was performed in an automated thermocycler (Thermo Electron, Waltham, MA, USA) under the following PCR program: initial denaturation step at $94^{\circ} \mathrm{C}$ for $5 \mathrm{~min}$, fol- 
Effects of MC-LR on gut microflora

lowed by 30 cycles of $1 \mathrm{~min}$ at $94^{\circ} \mathrm{C}, 1 \mathrm{~min}$ at $65^{\circ} \mathrm{C}$ (decreasing $1^{\circ} \mathrm{C}$ every cycle to a final of $55^{\circ} \mathrm{C}$ ), 1 min at $72^{\circ} \mathrm{C}$, and followed by a final $10 \mathrm{~min}$ extension step at $72^{\circ} \mathrm{C}$.

DGGE was performed as described by Muyzer on a Universal Mutation Detection System (Bio-Rad, Hercules, CA, USA) with an $8 \%(\mathrm{w} / \mathrm{v})$ polyacrylamide gel (37.5:1 acrylamide: bisacrylamide) containing a 40\%$55 \%$ gradient of urea and formamide (100\% denaturant contained $7 \mathrm{M}$ urea and $40 \%$ deionized formamide) (Muyzer et al., 1993). The PCR products of each sample were loaded on the gel and run at $110 \mathrm{~V}$ for $10 \mathrm{hr}$ at $60^{\circ} \mathrm{C}$ in a $1 \times$ TAE running buffer. After staining with 1:10000 diluted GelRed solution for $30 \mathrm{~min}$, the gels were viewed and photographed under UV illumination in a Bio Image System (Gene Genius, Syngene, Frederick, MD, USA).

\section{Sequencing of DGGE bands}

DGGE bands were excised and eluted in $40 \mu \mathrm{L}$ of sterile $\mathrm{ddH}_{2} \mathrm{O}$ and stored at $4^{\circ} \mathrm{C}$ overnight. The eluted DNA was re-amplified with the primers 341F (without a GC clamp) and 518R described above. PCR products were purified by Gel Extraction Kit (Omega Biotek Inc., Norcross, GA, USA) and then cloned into pMD18-T plasmid vector (Takara Bio Inc., Otsu, Shiga, Japan). Positive clones of each band were collected and sequenced.

\section{Nucleotide Sequence Accession Number}

The partial sequences of $16 \mathrm{~S}$ rRNA genes have been deposited in the NCBI database under Accession No. KJ766005 - KJ766109.

\section{Data analysis}

Blast of sequences was performed based on NCBI and the ribosomal database project (RDP), and phylogenetic analysis was performed with Clustal W and Mega 4.1 using the neighbor-joining method. Digitalized analyses were performed with Quantity One (version 4.6.2) by detecting the bands and reporting the intensity matrices of DGGE profiles. The relative intensity of each band in a lane was assumed to be the relative abundance of its corresponding phylotype in a sample (Fromin et al., 2002; Lyautey et al., 2005). The relative abundance data were submitted to Metastats software to detect the bands which have significantly different abundances between the two groups $(\mathrm{P}<0.05, \mathrm{n}=6)$. The bacterial diversity in the three intestinal regions of control and MC-LR-treated samples was analyzed through the Shannon index $\left(\mathrm{H}_{\mathrm{b}}{ }^{\prime}\right)$ by the equation $\mathrm{H}_{\mathrm{b}}{ }^{\prime}=-\sum_{i=1}^{N} P i \ln P i$, where $\mathrm{P}_{\mathrm{i}}$ is the relative intensity of the $i$ th band and $\mathrm{N}$ is the total number of bands in each lane of DGGE (Zhang et al., 2007). Pielou's evenness index $\left(J^{\prime}\right)$ was calculated by the equation $J^{\prime}=\mathrm{H}_{\mathrm{b}}{ }^{\prime} / \ln \mathrm{N}$ to reflect the uniformity of bacterial species distribution. Clustering analyses of DGGE profiles were performed with the NTSYS program version $2.10 \mathrm{e}$ (Exeter Software, Setauket, NY, USA) by scoring the presence or absence (1 or 0) of DGGE bands. The clustering algorithms used to calculate the dendrogram was an unweighted pair group method with arithmetic averages (UPGMA) (Liu et al., 2012).

\section{RESULTS}

\section{Animal health status}

Till the end of the animal experiment, no mice died of MC-LR toxicity. Meanwhile, no significant difference of bodyweight gain was found between the control and $\mathrm{MC}$ LR-treated groups.

\section{Variation of microbial community diversity in different gut regions after MC-LR treatment}

The profiles of microflora in the jejuno-ileum, caecum and colon were examined by PCR-DGGE analysis (Fig. 1). Theoretically, each DGGE band was considered to be a single operational taxonomic unit (OTU), and the banding pattern reflects a community's species richness and diversity (Gafan and Spratt, 2005). The comparison of microbial community composition (diversity and evenness) between MC-LR-treated samples and the controls were analyzed with the independent samples $t$-test (Table 1). Compared with the controls, the microbial species richness (number of DGGE bands) of MC-LR-treated samples was significantly increased in the caecum $(P<0.01)$ and colon $(P<0.05)$, and the microbial diversity (Shannon's index) in caecum was significantly increased in MC-LR-treated group $(P<0.05)$. However, in jejuno-ileum, no significant differences were found in species richness (band number) as well as diversity index between the control and MC-LR treatment samples. These results indicated that gut microbial species richness and diversity increased significantly after MC-LR treatment, but the effect was varied in different gut regions. Meanwhile, the evenness (Pielou's index) of gut microflora was not affected by MC-LR, as the Pielou's index between control and MC-LR treated groups had no significant difference $(P>0.05)$ in all three gut regions.

\section{Similarity analysis of DGGE profiles}

To analyze the effects of MC-LR on the microbial compositions in the jejuno-ileum, caecum and colon, the 


\section{J. Chen et al.}

\section{IL}

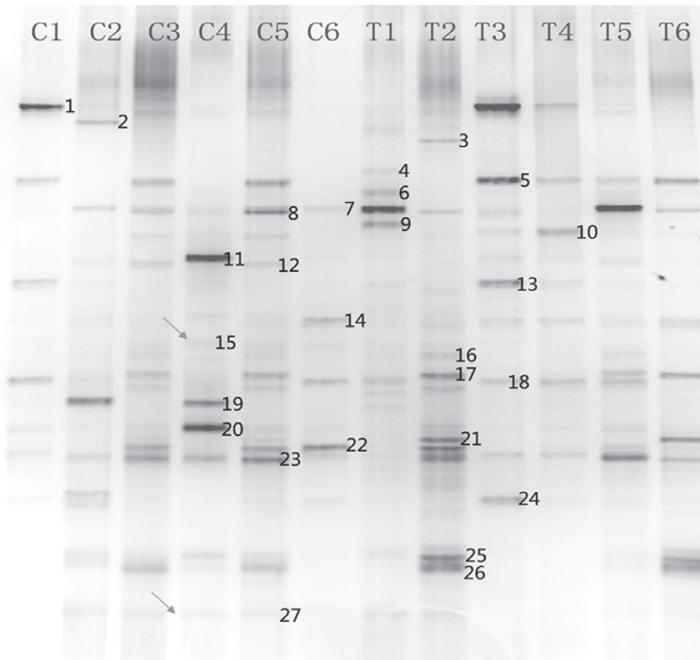

\section{CA}

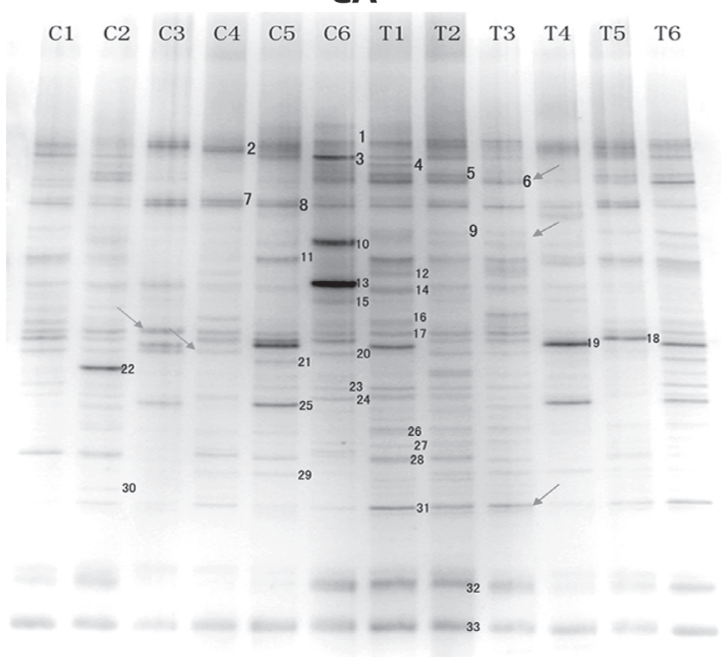

CO

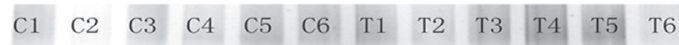

Fig. 1. The DGGE patterns of 16S rDNA V3 region of bacteria in the jejuno-ileum (IL), caecum (CA) and colon (CO). C1-C6: control samples; T1-T6: MC-LR-treated samples. Numbers were assigned to bands based on electrophoretic mobility. Bands signals decreased significantly after MC-LR treatment were pointed out with right arrows $(\searrow$ ) on the left, and the increased ones were pointed out with left arrows $(\swarrow)$ on the right.

Table 1. Diversity analysis of microflora in the jejuno-ileum, caecum and colon between control and MC-LR-treated samples.

\begin{tabular}{|c|c|c|c|c|c|c|}
\hline \multirow{2}{*}{ Diversity index ${ }^{a}$} & \multicolumn{2}{|c|}{ Jejuno-ileum } & \multicolumn{2}{|c|}{ Caecum } & \multicolumn{2}{|c|}{ Colon } \\
\hline & Control & Treated & Control & Treated & Control & Treated \\
\hline Species richness & $18 \pm 2.08$ & $20 \pm 2.21$ & $21 \pm 0.63$ & $29.8 \pm 0.31^{b}$ & $26.3 \pm 0.49$ & $30.8 \pm 1.33^{c}$ \\
\hline Shannon-Wiener & $2.19 \pm 0.25$ & $2.72 \pm 0.19$ & $2.50 \pm 0.05$ & $2.78 \pm 0.09 c$ & $2.72 \pm 0.06$ & $2.80 \pm 0.10$ \\
\hline Pielou's & $0.76 \pm 0.06$ & $0.76 \pm 0.06$ & $0.82 \pm 0.02$ & $0.82 \pm 0.03$ & $0.83 \pm 0.04$ & $0.81 \pm 005$ \\
\hline
\end{tabular}

aData are shown as Mean \pm S.D.

${ }^{\mathrm{b}} P<0.001$ between control and treated group.

${ }^{\mathrm{c}} P<0.05$ between control and treated group. 
Effects of MC-LR on gut microflora
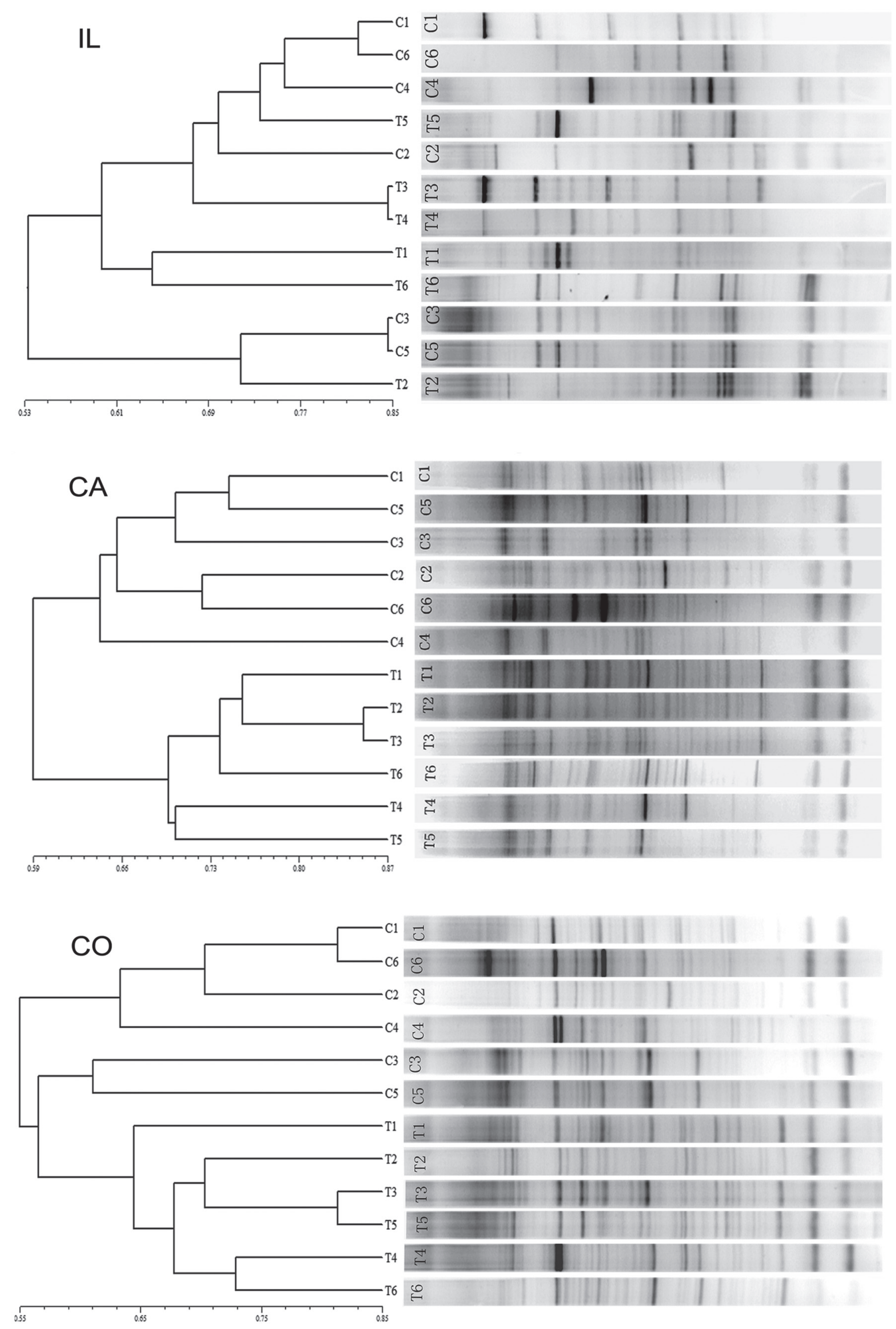

Fig. 2. UPGMA clustering dendrogram representing the similarity of microbiota in the jejuno-ileum (IL), caecum (CA) and colon (CO). C1-C6, control samples; T1-T6, MC-LR-treated samples. 
dendrograms of the DGGE profiles, which could indicate band pattern similarity between samples, were constructed by UPGMA clustering method (Fig. 2). Samples with higher similarity between DGGE band patterns cluster more strongly together. As shown in Fig. 2, in the jejunoileum, samples of the control group and the MC-LR-treated group were randomly clustered together. In the caecum, samples of the control group and the MC-LR-treated group were distinctly separated into two defined clusters, and the value of similarity between the two groups was $59 \%$. Samples from the colon were grouped into 3 clusters. The MC-LR-treated group samples formed a defined cluster and separated with control group samples at the $64 \%$ similarity level. The clustering results showed that MC-LR induced significant shifts of microbial community in the caecum and colon, however, the effects of MCLR on the jejuno-ileum microbiota were not obvious.

\section{Compositions of microbial communities in different gut regions}

27,33 and 45 sequences were obtained from the jejuno-ileum, caecum and colon in the DGGE profiles, respectively. Almost all matched bacteria of the sequences were assigned to 4 phyla: Firmicutes, Bacteroidetes, Proteobacteria and TM7. The phylogenetic trees of all the obtained sequences were generated and showed that the majority of the sequences were assigned to Clostridia in phylum Firmicutes (Supplementary Fig. 1). The bacterial compositions of the three gut regions were estimated at the family level according to the proportions of sequenced clones (Fig. 3). Generally, in the jejuno-ileum, the microbial sequences affiliated with 9 families in 7 classes; sequences in the caecum fell into 5 families in 4 classes; sequences in the colon assigned to 9 families in 5 classes. Two families were common in all three regions; Porphyromonadaceae in the Bacteroidetes phylum and Lachnospiraceae from Clostridia in the Firmicutes phylum. Porphyromonadaceae was the most abundant $(29.6 \%)$ in the jejuno-ileum, whereas Lachnospiraceae was dominant in the caecum and colon with proportions of $66.7 \%$ and $55.6 \%$, respectively.

\section{Microbial shifts after MC-LR treatment}

To find out the significantly shifted microbes after MC-



Fig. 3. Microbial compositions in jejuno-ileum (IL), caecum (CA) and colon (CO).

\author{
$\therefore$ unclassified \\ "Ruminococcaceae \\ Helicobacteraceae \\ ㅅ: Rikenellaceae \\ \% prevotellaceae \\ II Bacteroidaceae \\ - streptococcaceae \\ $\therefore$ Lactobacillaceae \\ Erysipelotrichaceae \\ II Bacillales_incertae sedis XI \\ Xanthomonadaceae \\ -Enterobacteriaceae \\ "Desulfovibrionaceae \\ Lachnospiraceae
}

a porphyromonadaceae 
Effects of MC-LR on gut microflora

Table 2. Taxonomic identities of the shifted microbes after MC-LR treatment.

\begin{tabular}{llllc}
\hline DGGE bands & \multicolumn{1}{c}{ Class } & \multicolumn{1}{c}{ Family } & \multicolumn{1}{c}{ Closest relative (accession number) } & Similarity (\%) \\
\hline Decreased & & & & \\
IL15 & Clostridia & Lachnospiraceae & Clostridium XlVa sp. (AY992008) & 100 \\
IL27 & Clostridia & Lachnospiraceae & Uncultured bacterium (EF098562) & 96 \\
CA17 & Clostridia & Ruminococcaceae & Oscillospira guilliermondii (AB040498) & 100 \\
CA20 & Clostridia & Lachnospiraceae & Uncultured bacterium (EU508203) & 98 \\
CO22 & Clostridia & Lachnospiraceae & Uncultured bacterium (EF407306) & 100 \\
CO23 & Clostridia & Lachnospiraceae & Clostridium XlVa sp. (AY991138) & 100 \\
CO28 & Clostridia & Lachnospiraceae & Eubacterium plexicaudatum (AF157054) & 96 \\
CO45 & Clostridia & Lachnospiraceae & Uncultured bacterium (EF406499) & 98 \\
\hline Increased & & & & 100 \\
CA6 & Bacteroidia & Porphyromonadaceae & Barnesiella sp. (AY989935) & 100 \\
CA9 & Clostridia & Lachnospiraceae & Uncultured bacterium (EF098280) & 100 \\
CA31 & Bacteroidia & Porphyromonadaceae & Barnesiella sp. (AY989959) & 97 \\
CO32 & Bacteroidia & Prevotellaceae & Prevotella sp. (DQ014597) & 100 \\
CO35 & Clostridia & Ruminococcaceae & Flavonifractor plautii sp.(GQ449176) & 100 \\
CO38 & Clostridia & Lachnospiraceae & Uncultured bacterium (AY991711) & 100 \\
CO43 & Bacteroidia & Porphyromonadaceae & Barnesiella sp. (AY989959) & \\
\hline
\end{tabular}

LR treatment, the relative abundance matrixes, which were obtained according to the gray value of each band in the DGGE profiles, were submitted to Metastats software on the 0.05 significance level. In total, 17 sequences were detected to have significant differences between the control and MC-LR-treated samples, among which 2 sequences were from the jejuno-ileum, 5 sequences were from the caecum and 8 sequences were from the colon (Fig. 1 and Table 2). Moreover, all of the significantly decreased sequences were assigned to the class Clostridia in phylum Firmicutes (Table 2), and the majority of them derived from the Lachnospiraceae family, except for one sequence belonging to the Ruminococcaceae family. The significantly increased microbes were detected only in the ceacum and colon, and the increased ones were from diverse families including Porphyromonadaceae, Lachnospiraceae, Ruminococcaceae and Prevotellaceae in both Bacteriodetes and Firmicutes phyla. Above all, it should be mentioned that the sequences CA31 and CO43 that have $100 \%$ similarity to Barnesiella from Porphyromonadaceae in the Bacteroidetes phylum increased significantly.

\section{DISCUSSION}

Trillions of microbes live in symbiosis with the host in the intestinal tract, which act as an immune and metabolic "organ" interacting extensively with the host. In the mouse, the gut microbiota plays an important role in energy and nutrition absorption (Hooper et al., 2002; Turnbaugh et al., 2006), intestinal epithelia growth (Guarner and Malagelada, 2003), immune system maturation and defense against pathogenic bacteria infection (Hooper et al., 2012). Dysbiosis of gut microbota has been associated with various intestinal and hepatic diseases, including inflammatory bowel disease (IBD) (Chassard et al., 2012), colon cancer (Scanlan et al., 2008), nonalcoholic steatohepatitis (NASH) (HenaoMejia et al., 2012; Aron-Wisnewsky et al., 2013) and liver cirrhosis (Goel et al., 2014). Disorder of bacteria composition in the gut may indicate the risk of some diseases, and hence can be used as an indicator of illness and an aid for health assessment (Sekirov et al., 2010; Qin et al., 2012).

In our experiment, we mimicked the natural way of MC-LR intoxication in a mice model to evaluate the toxic effects of MC-LR on gut microbes and further on human health. The main route of exposure to MCs in human is through water intake, and chronic exposure to sublethal concentrations of MCs is the most common intoxication in animals and humans (Nicholson et al., 2005; Sedan et al., 2010; Zeller et al., 2012). Therefore, we conducted a 
sub-chronic exposure of MC-LR by oral gavage to mice at $200 \mathrm{ug} / \mathrm{kg}$ body weight $\left(\sim 1 / 55 \mathrm{LD}_{50}\right)$ for 3 months at $48 \mathrm{hr}$ intervals.

The density, diversity and compositions of microbiota vary along the intestinal tract, which was related to different environments in different intestinal regions. Considering this, we revealed the microbial topographic distribution in $\mathrm{BALB} / \mathrm{c}$ mice, and specifically examined the effects of MC-LR on the microbes in mice's jejuno-ileum, caecum and colon. The jejuno-ileum is part of the small intestine, while the caecum and colon belong to the large intestine. Previous studies on topographical intestinal biochemistry and metabolomics have revealed that the metabolic fingerprints varied among different intestinal regions, and the greatest differences were present between the small and large intestines (Wang et al., 2005b, 2007, 2008). Consistent with previous studies, the microbial density increased along the intestinal tract (Mackie et al., 1999; Wang et al., 2005a; Goel et al., 2014). The band number and diversity index were the lowest in the jejuno-ileum, and the colon harbored the most microbes among the three intestinal regions. Moreover, the microbial composition in the jejuno-ileum was quite different from that in the caecum and colon. In mice, as well as in humans, Firmicutes and Bacteroidetes are two main phyla of bacteria in the gut microbiota. In the present study, we found that distributions of the two phyla varied markedly between the jejuno-ileum and the other two regions. While Firmicutes, especially Lachnospiraceae, was absolutely predominant in caecum and colon, the Porphyromonadaceae in Bacteroidetes was the most ubiquitous in the jejuno-ileum.

It is reported that MC-LR can induce oxidative stress on prokaryotes and cause a series changes in physiological and biochemical characteristics in prokaryotic cells (Yang et al., 2009). Our recent study has shown that MCLR can significantly enrich the microbial genes involved in protection against oxidative stress and degradation of carbon compounds in rat gut (Lin et al., 2015). In the current study, we examined the effects of MC-LR on the gut microbes in different gut regions of mice. We found that MC-LR could significantly affect the gut microflora in mice, and as we expected, the effects varied in different gut regions. According to the cluster analysis, MC-LR induced significant shifts of microbial compositions in the caecum and colon. However, no significant variation was detected in the jejuno-ileum. Host disease as well as some toxic agents has been shown to decrease gut microbial diversity (Ling et al., 2014; Stevenson et al., 2014; Wu et al., 2014). Unexpectedly, in our study, the hepatotoxic MC-LR increased the gut microbial diversity. We observed significantly increased species richness in the caecum and colon, and significantly increased microbial diversity in the caecum after MC-LR exposure. We failed to give a definite explain with our present study. However, Liu et al. (2012) found an increased diversity of microbial composition in liver cirrhosis patients, and they speculated that it might be related to bacterial overgrowth in chronic liver disease.

MC-LR induced significant shifts of some microbes. The decreased microbes were Lachnospiraceae, expect for an Oscillibacter in Ruminococcaceae. And, the increase of Barnesiella in the caecum and colon of mice was most obvious. Interestingly, a similar microbial shift was shown in a study of diabetic mice, which found $28 \%$ reduction in the Lachnospiraceae family and a $54 \%$ reduction in the Oscillibacter genus in diabetic versus nondiabetic mice (McDermid, 2012). At the meantime, previous studies also found increased Barnesiella in dietary gluten induced hyperglycemia and Type 1 diabetes of non-obese diabetic mice (Marietta et al., 2013), and in NASH-prone mice which developed hepatic macrovesicular steatosis (Le Roy et al., 2013). Most notably, it should be mentioned that the mice in the MC-LR-treated group did show signs of NASH (unpublished data). As a potent hepatotoxin, MC-LR's primary target organ is the liver and can induce cell apoptosis, hepatitis and carcinoma (Chen et al., 2005). Moreover, enterohepatic cycling make the liver and the gut closely related in terms of MC-LR absorption, transportation and pathogenic process (Liu et al., 2012). It is reasonable to speculate that the alterations of gut microbiota induced by MC-LR exposure might play an important role in induction and promotion of liver damage progression of MC-LR. Further studies should be taken to verify this speculation.

In summary, subchronic oral exposure to MC-LR at a dose of $200 \mu \mathrm{g} / \mathrm{kg}$ body weight has toxic effects on the gut microbes of mice. We observed different effects of MC-LR on microbes residing in the jejuno-ileum, caecum and colon. The structures of microflora in the caecum and colon are significantly altered after MC-LR exposure, while that in the jejuno-ileum is not. The shifted structure of gut microflora may cause metabolic disorders and diseases in mice. This study profiled the topographical distribution of BALB/c mice gut microbes, and revealed the different effects of MC-LR on the gut microflora in different gut regions. This research helps understanding of the effects of MC-LR in the aspect of gut microbiota, which adds new information for the assessment of MCLR's safety to human health. 
Effects of MC-LR on gut microflora

\section{ACKNOWLEDGMENTS}

This work was supported by the National Natural Science Foundation of China (31322013; 31070457).

Conflict of interest---- The authors declare that there is no conflict of interest.

\section{REFERENCES}

Aron-Wisnewsky, J., Gaborit, B., Dutour, A. and Clement, K. (2013): Gut microbiota and non-alcoholic fatty liver disease: new insights. Clin. Microbiol. Infect., 19, 338-348.

Bates, J.M., Akerlund, J., Mittge, E. and Guillemin, K. (2007): Intestinal alkaline phosphatase detoxifies lipopolysaccharide and prevents inflammation in zebrafish in response to the gut microbiota. Cell Host Microbe, 2, 371-382.

Botha, N., Van de Venter, M., Downing, T.G., Shephard, E.G. and Gehringer, M.M. (2004): The effect of intraperitoneally administered microcystin-LR on the gastrointestinal tract of $\mathrm{Balb} / \mathrm{c}$ mice. Toxicon, 43, 251-254.

Chassard, C., Dapoigny, M., Scott, K.P., Crouzet, L., Del'homme, C., Marquet, P., Martin, J.C., Pickering, G., Ardid, D., Eschalier, A., Dubray, C., Flint, H.J. and Bernalier-Donadille, A. (2012): Functional dysbiosis within the gut microbiota of patients with constipated-irritable bowel syndrome. Aliment. Pharmacol. Ther., 35, 828-838.

Chen, T., Wang, Q., Cui, J., Yang, W., Shi, Q., Hua, Z., Ji, J. and Shen, P. (2005): Induction of apoptosis in mouse liver by microcystin-LR: a combined transcriptomic, proteomic, and simulation strategy. Mol. Cell. Proteomics, 4, 958-974.

Codd, G.A. (2000): Cyanobacterial toxins, the perception of water quality, and the prioritisation of eutrophication control. Ecol. Eng., 16, 51-60.

Dawson, R.M. (1998): The toxicology of microcystins. Toxicon, $\mathbf{3 6}$, 953-962.

Druart, C., Neyrinck, A.M., Vlaeminck, B., Fievez, V., Cani, P.D. and Delzenne, N.M. (2014): Role of the lower and upper intestine in the production and absorption of gut microbiota-derived PUFA metabolites. PLoS One, 9, e87560.

Faber, K.N., Müller, M. and Jansen, P.L. (2003): Drug transport proteins in the liver. Adv. Drug Deliv. Rev., 55, 107-124.

Fastner, J., Codd, G.A., Metcalf, J.S., Woitke, P., Wiedner, C. and Utkilen, H. (2002): An international intercomparison exercise for the determination of purified microcystin-LR and microcystins in cyanobacterial field material. Anal. Bioanal. Chem., 374, 437-444.

Fromin, N., Hamelin, J., Tarnawski, S., Roesti, D., JourdainMiserez, K., Forestier, N., Teyssier-Cuvelle, S., Gillet, F., Aragno, M. and Rossi, P. (2002): Statistical analysis of denaturing gel electrophoresis (DGE) fingerprinting patterns. Environ. Microbiol., 4, 634-643.

Gafan, G.P. and Spratt, D.A. (2005): Denaturing gradient gel electrophoresis gel expansion (DGGEGE) -an attempt to resolve the limitations of co-migration in the DGGE of complex polymicrobial communities. FEMS Microbiol. Lett., 253, 303-307.

Goel, A., Gupta, M. and Aggarwal, R. (2014): Gut microbiota and liver disease. J. Gastroenterol. Hepatol., 29, 1139-1148.

Guarner, F. and Malagelada, J.-R. (2003): Gut flora in health and disease. Lancet, 361, 512-519.
Henao-Mejia, J., Elinav, E., Jin, C., Hao, L., Mehal, W.Z., Strowig, T., Thaiss, C.A., Kau, A.L., Eisenbarth, S.C., Jurczak, M.J., Camporez, J.P., Shulman, G.I., Gordon, J.I., Hoffman, H.M. and Flavell, R.A. (2012): Inflammasome-mediated dysbiosis regulates progression of NAFLD and obesity. Nature, 482, 179-185.

Hooper, L.V., Littman, D.R. and Macpherson, A.J. (2012): Interactions between the microbiota and the immune system. Science, 336, 1268-1273.

Hooper, L.V., Midtvedt, T. and Gordon, J.I. (2002): How host-microbial interactions shape the nutrient environment of the mammalian intestine. Annu. Rev. Nutr., 22, 283-307.

Humpage, A.R., Hardy, S.J., Moore, E.J., Froscio, S.M. and Falconer, I.R. (2000): Microcystins (cyanobacterial toxins) in drinking water enhance the growth of aberrant crypt foci in the mouse colon. J. Toxicol. Environ. Health Part A, 61, 155-165.

Ito, E., Kondo, F. and Harada, K.-I. (2000): First report on the distribution of orally administered microcystin-LR in mouse tissue using an immunostaining method. Toxicon, 38, 37-48.

Jiao, J., Lu, Q., Tan, Z., Guan, L., Zhou, C., Tang, S. and Han, X. (2014a): In vitro evaluation of effects of gut region and fiber structure on the intestinal dominant bacterial diversity and functional bacterial species. Anaerobe, 28, 168-177.

Jiao, J., Wang, P., He, Z., Tang, S., Zhou, C., Han, X., Wang, M., Wu, D., Kang, J. and Tan, Z. (2014b): In vitro evaluation on neutral detergent fiber and cellulose digestion by post-ruminal microorganisms in goats. J. Sci. Food Agric., 94, 1745-1752.

Lürling, M. and Faassen, E.J. (2013): Dog poisonings associated with a Microcystis aeruginosa bloom in the Netherlands. Toxins, 5, 556-567.

Le Roy, T., Llopis, M., Lepage, P., Bruneau, A., Rabot, S., Bevilacqua, C., Martin, P., Philippe, C., Walker, F., Bado, A., Perlemuter, G., Cassard-Doulcier, A.M. and Gérard, P. (2013): Intestinal microbiota determines development of non-alcoholic fatty liver disease in mice. Gut, 62, 1787-1794.

Li, H., Cai, Y., Xie, P., Chen, J., Hao, L., Li, G. and Xiong, Q. (2012): Identification and expression profile of Id 1 in bighead carp in response to microcystin-LR. Environ. Toxicol. Pharmacol., 34, 324-333.

Li, L. and Xie, P. (2009): Hepatic histopathological characteristics and antioxidant response of phytoplanktivorous silver carp intraperitoneally injected with extracted microcystins. Biomed. Environ. Sci., 22, 297-302.

Lin, J., Chen, J., He, J., Chen, J., Yan, Q., Zhou, J. and Xie, P. (2015): Effects of microcystin-LR on bacterial and fungal functional genes profile in rat gut. Toxicon, 96, 50-56.

Ling, Z.X., Liu, X., Jia, X.Y., Cheng, Y.W., Luo, Y.Q., Yuan, L. Wang, Y.Z., Zhao, C.N., Guo, S. and Li, L.J. (2014): Impacts of infection with different toxigenic Clostridium difficile strains on faecal microbiota in children. Sci. Rep., 4, 7485.

Liu, J., Wu, D., Ahmed, A., Li, X., Ma, Y., Tang, L., Mo, D., Ma, Y. and Xin, Y. (2012): Comparison of the gut microbe profiles and numbers between patients with liver cirrhosis and healthy individuals. Curr. Microbiol., 65, 7-13.

Liu, L. and Xie, P. (2014): Effects of microcystin-LR on the digestive enzyme activity of intestinal tract in BALB/c mice. Acta Hydrobiologica Sinica (in Chinese), 38, 533-539.

Loh, G. and Blaut, M. (2012): Role of commensal gut bacteria in inflammatory bowel diseases. Gut Microbes, 3, 544-555.

Lyautey, E., Lacoste, B., Ten-Hage, L., Rols, J.-L. and Garabetian, F. (2005): Analysis of bacterial diversity in river biofilms using 16S rDNA PCR-DGGE: methodological settings and fingerprints interpretation. Water Res., 39, 380-388. 
Mackie, R.I., Sghir, A. and Gaskins, H.R. (1999): Developmental microbial ecology of the neonatal gastrointestinal tract. Am. J. Clin. Nutr., 69, 1035s-1045s.

Marietta, E.V., Gomez, A.M., Yeoman, C., Tilahun, A.Y., Clark, C.R., Luckey, D.H., Murray, J.A., White, B.A., Kudva, Y.C. and Rajagopalan, G. (2013): Low incidence of spontaneous type 1 diabetes in non-obese diabetic mice raised on gluten-free diets is associated with changes in the intestinal microbiome. PLoS One, 8, e78687.

McDermid, E. (2012): Gut microbiota may dictate vulnerability to diet-induced diabetes. Gut, 61, 543-553.

Messori, S., Trevisi, P., Simongiovanni, A., Priori, D. and Bosi, P. (2013): Effect of susceptibility to enterotoxigenic Escherichia coli F4 and of dietary tryptophan on gut microbiota diversity observed in healthy young pigs. Vet. Microbiol., 162, 173-179.

Metcalf, J.S., Bell, S.G. and Codd, G.A. (2001): Colorimetric immuno-protein phosphatase inhibition assay for specific detection of microcystins and nodularins of cyanobacteria. Appl. Environ. Microbiol., 67, 904-909.

Muyzer, G., De Waal, E.C. and Uitterlinden, A.G. (1993): Profiling of complex microbial populations by denaturing gradient gel electrophoresis analysis of polymerase chain reaction-amplified genes coding for 16S rRNA. Appl. Environ. Microbiol., 59, 695700.

Nicholson, J.K., Holmes, E. and Wilson, I.D. (2005): Gut microorganisms, mammalian metabolism and personalized health care. Nat. Rev. Microbiol., 3, 431-438.

Nishiwaki-Matsushima, R., Ohta, T., Nishiwaki, S., Suganuma, M., Kohyama, K., Ishikawa, T., Carmichael, W.W. and Fujiki, H. (1992): Liver tumor promotion by the cyanobacterial cyclic peptide toxin microcystin-LR. J. Cancer Res. Clin. Oncol., 118, 420-424.

Pouria, S., de Andrade, A., Barbosa, J., Cavalcanti, R.L., Barreto, V.T., Ward, C.J., Preiser, W., Poon, G.K., Neild, G.H. and Codd, G.A. (1998): Fatal microcystin intoxication in haemodialysis unit in Caruaru, Brazil. Lancet, 352, 21-26.

Puhl, N.J., Uwiera, R.R.E., Yanke, L.J., Selinger, L.B. and Inglis, G.D. (2012): Antibiotics conspicuously affect community profiles and richness, but not the density of bacterial cells associated with mucosa in the large and small intestines of mice. Anaerobe, 18, 67-75.

Qin, J., Li, Y., Cai, Z., Li, S., Zhu, J., Zhang, F., Liang, S., Zhang, W., Guan, Y., Shen, D., Peng, Y., Zhang, D., Jie, Z., Wu, W., Qin, Y., Xue, W., Li, J., Han, L., Lu, D., Wu, P., Dai, Y., Sun, X., Li, Z., Tang, A., Zhong, S., Li, X., Chen, W., Xu, R., Wang, M., Feng, Q., Gong, M., Yu, J., Zhang, Y., Zhang, M., Hansen, T., Sanchez, G., Raes, J., Falony, G., Okuda, S., Almeida, M., LeChatelier, E., Renault, P., Pons, N., Batto, J.M., Zhang, Z., Chen, H., Yang, R., Zheng, W., Li, S., Yang, H., Wang, J., Ehrlich, S.D., Nielsen, R., Pedersen, O., Kristiansen, K. and Wang, J. (2012): A metagenome-wide association study of gut microbiota in type 2 diabetes. Nature, 490, 55-60.

Runnegar, M.T., Gerdes, R.G. and Falconer, I.R. (1991): The uptake of the cyanobacterial hepatotoxin microcystin by isolated rat hepatocytes. Toxicon, 29, 43-51.

Scanlan, P.D., Shanahan, F., Clune, Y., Collins, J.K., O'Sullivan, G.C., O'Riordan, M., Holmes, E., Wang, Y. and Marchesi J.R. (2008): Culture-independent analysis of the gut microbiota in colorectal cancer and polyposis. Environ. Microbiol., 10, 789798.

Sedan, D., Andrinolo, D., Telese, L., Giannuzzi, L., de Alaniz, M.J. and Marra, C.A. (2010): Alteration and recovery of the antioxidant system induced by sub-chronic exposure to microcystin-LR in mice: its relation to liver lipid composition. Toxicon, 55, 333342.

Sekirov, I., Russell, S.L., Antunes, L.C.M. and Finlay, B.B. (2010): Gut microbiota in health and disease. Physiol. Rev., 90, 859904.

Stevenson, T.J., Buck, C.L. and Duddleston, K.N. (2014): Temporal dynamics of the cecal gut microbiota of juvenile arctic ground squirrels: a strong litter effect across the first active season. Appl. Environ. Microbiol., 80, 4260-4268.

Tian, Y., Zhang, L. Wang, Y. and Tang, H. (2011): Age-related topographical metabolic signatures for the rat gastrointestinal contents. J. Proteome Res., 11, 1397-1411.

Turnbaugh, P.J., Ley, R.E., Mahowald, M.A., Magrini, V., Mardis, E.R. and Gordon, J.I. (2006): An obesity-associated gut microbiome with increased capacity for energy harvest. Nature, $\mathbf{4 4 4}$, 1027-1131.

Wang, M., Ahrné, S., Jeppsson, B. and Molin, G. (2005a): Comparison of bacterial diversity along the human intestinal tract by direct cloning and sequencing of $16 \mathrm{~S}$ rRNA genes. FEMS Microbiol. Ecol., 54, 219-231.

Wang, Y., Cloarec, O., Tang, H., Lindon, J.C., Noimes, E., Kochhar, S. and Nicholson, J.K. (2008): Magic angle spinning NMR and $1 \mathrm{H}-31 \mathrm{P}$ heteronuclear statistical total correlation spectroscopy of intact human gut biopsies. Anal. Chem., 80, 10581066.

Wang, Y., Holmes, E., Comelli, E.M., Fotopoulos, G., Dorta, G., Tang, H., Rantalainen, M.J., Lindon, J.C., Corthesy-Theulaz, I.E., Fay, L.B., Kochhar, S. and Nicholson, J.K. (2007): Topographical variation in metabolic signatures of human gastrointestinal biopsies revealed by high-resolution magic-angle spinning 1H NMR spectroscopy. J. Proteome Res., 6, 3944-3951.

Wang, Y.L., Tang, H.R., Holmes, E., Lindon, J.C., Turini, M.E., Sprenger, N., Bergonzelli, G., Fay, L.B., Kochhar, S. and Nicholson, J.K. (2005b): Biochemical characterization of rat intestine development using high-resolution magic-angle-spinning $1 \mathrm{H}$ NMR spectroscopy and multivariate data analysis. J. Proteome. Res., 4, 1324-1329.

Wu, B., Cui, H., Peng, X., Pan, K., Fang, J., Zuo, Z., Deng, J., Wang, X. and Huang, J. (2014): Toxicological effects of dietary nickel chloride on intestinal microbiota. Ecotoxicol. Environ. Saf., 109, 70-76.

Yan, W., Zhou, Y., Yang, J., Li, S., Hu, D., Wang, J., Chen, J. and Li, G. (2012): Waterborne exposure to microcystin-LR alters thyroid hormone levels and gene transcription in the hypothalamic-pituitary-thyroid axis in zebrafish larvae. Chemosphere, 87, 1301-1307.

Yang, C.Y., Liu, S.J., Zhou, S.W. Xia, C.H. and Liu, Y.D. (2009): Advance in study on the ecotoxicological effect of microcystins against microorganisms. Asian J. Ecotox. (in Chinese), 4, 602-608.

Zeller, P., Quenault, H., Huguet, A., Blanchard, Y. and Fessard, V. (2012): Transcriptomic comparison of cyanotoxin variants in a human intestinal model revealed major differences in oxidative stress response: Effects of MC-RR and MC-LR on Caco-2 cells. Ecotox. Environ. Safe., 82, 13-21.

Zhang, D., Xie, P., Liu, Y., Chen, J. and Wen, Z. (2009): Spatial and temporal variations of microcystins in hepatopancreas of a freshwater snail from Lake Taihu. Ecotox. Environ. Safe., 72, 466472.

Zhang, M., Liu, B., Zhang, Y., Wei, H., Lei, Y. and Zhao, L. (2007): Structural shifts of mucosa-associated lactobacilli and Clostridium leptum subgroup in patients with ulcerative colitis. J. Clin. Microbiol., 45, 496-500. 